\title{
Mémoire procédurale et processus cognitifs: application de règles en morphologie, syntaxe et calcul arithmétique
}

\author{
Joël Macoir\& Marion Fossard \\ Programme de maitrise en orthophonie - Faculté de médecine - Université Laval; Québec (Qué); Canada \\ Centre de Recherche Université Laval Robert-Giffard - Axe recherche évaluative et clinique \\ joel.macoir@rea.ulaval.ca
}

\section{Introduction}

Pendant très longtemps, la caractérisation des processus normaux et pathologiques de traitement des informations linguistiques a été effectuée de manière totalement isolée de celle relative aux autres fonctions cognitives. Selon cette approche traditionnelle, on considère que le langage est différent de la mémoire, de l'attention, des fonctions exécutives, etc., et doit donc être appréhendé séparément du reste de la cognition. Bien que rassurante et pleinement justifiée sur le plan clinique, cette approche n'est cependant plus acceptable lorsqu'on s'intéresse aux processus cognitifs fondamentaux qui sous-tendent les habiletés linguistiques.

En effet, le langage, qui occupe une place centrale en cognition, est étroitement relié aux fonctions cognitives primaires (attention, mémoire de travail) ainsi qu'aux fonctions mentales supérieures telles que la mémoire. En fait, selon cette conception, une performance déficitaire, un comportement particulier ou une erreur spécifique peut certainement être de nature purement langagière mais peut aussi directement résulter d'une atteinte primaire non linguistique, telle qu'une atteinte de la mémoire procédurale.

Dans cet article, nous présenterons d'abord brièvement la conceptualisation générale du langage puis celle de la mémoire. Nous aborderons ensuite la question de la mémoire procédurale et de son interrelation avec les fonctions linguistiques par la présentation d'un modèle théorique et d'une illustration clinique. La conclusion nous permettra enfin de synthétiser les propos et de souligner l'importance de la prise en compte de la mémoire procédurale dans les approches diagnostiques et thérapeutiques des troubles acquis du langage.

\section{Conceptualisation linguistique et psycholinguistique du langage}

On trouve dans la plupart des modèles linguistiques une distinction formelle entre le lexique et la grammaire (Chomsky, 1995; S. Pinker, Jackendoff, R., 2005). Selon cette conception, les milliers de mots que connaît un individu sont encodés dans le lexique, composante linguistique dans laquelle sont encodées toutes les informations idiosyncrasiques relatives aux mots, incluant leur forme phonologique ainsi que des informations non prédictibles telles le nombre et la nature des arguments d'un verbe (ex., le verbe 'donner' requiert trois arguments syntaxiques: un sujet/agent, un objet direct/thème, et un objet indirect/bénéficiaire) ou les formes conjuguées de certains verbes (ex : 'aller'- 'va' - 'ira').) et adjectifs (ex., 'mou - molle') irréguliers.

Une très grande partie du langage est cependant sous-tendue par des opérations très régulières régies par l'autre composante principale du système linguistique, la grammaire. Cette composante réfère aux principes d'organisation de la langue, représentés sous la forme de règles spécifiant la combinaison des formes linguistiques (morphèmes et mots) pour la création d'un nombre infini de mots complexes, de syntagmes et de phrases. Dans le domaine morphologique par exemple, des règles grammaticales spécifient la combinaison de la racine verbale (ex., 'mange') avec les suffixes flexionnels de nombre et de temps ('il mangera'). 
Sur le plan cognitif, la plupart des modèles psycholinguistiques (Marcus, 2000; Marslen-Wilson \& Tyler, 1997; S. Pinker, 1999) proposent également une distinction entre lexique et grammaire. Ainsi, selon le modèle "words and rules" (S. Pinker, 1999), les mots sont encodés et récupérés dans le lexique mental dans les situations de compréhension et de production du langage. Selon ce modèle, le lexique mental est conceptualisé sous la forme d'une mémoire à long terme comprenant tous les mots dont la relation "son sens" est arbitraire tels que les racines des verbes et des adjectifs réguliers et les formes fléchies des verbes et adjectifs irréguliers. Les mots peuvent aussi être combinés via l'application de règles du langage qui sont par exemple requises pour la flexion des verbes et adjectifs réguliers, la dérivation et la syntaxe. Ainsi, la production ou la compréhension d'un verbe régulier requiert la récupération de la racine verbale dans le lexique mental et l'application de règles grammaticales de conjugaison dans la grammaire mentale. Pour un verbe irrégulier comme "aller" par contre, le processus de compréhension ou de production est limité à la récupération de la forme fléchie du verbe dans le lexique.

Bien que fonctionnellement indépendants, le lexique et la grammaire ne sont pas totalement isolés des autres fonctions cognitives et entretiennent des relations étroites avec la mémoire, une habileté cognitive composée de systèmes distincts et interconnectés (Schacter, Wagner, \& Buckner, 2000; Squire, Stark, \& Clark, 2004) dont certains sont responsables du maintien à long terme de l'information.

\section{Conceptualisation générale de la mémoire à long terme}

De manière très générale, la mémoire peut être conceptualisée comme une fonction mentale permettant à l'individu d'encoder, de retenir et de récupérer des connaissances ou des informations. Loin d'être une entité monolithique, la mémoire humaine, comme la plupart des processus cognitifs, est un système complexe, sous-tendu par diverses composantes spécialisées pour le traitement de différents types d'informations à court et à long terme. La notion de mémoire est d'ailleurs en pleine évolution et on assiste actuellement à une redéfinition du concept et à l'émergence de modèles intégratifs dans lesquels l'interrelation entre les différents systèmes mnésiques (mémoire de travail, mémoires à long terme) est prise en compte (ex., Eustache \& Desgranges, 2003).

Malgré ces nouvelles conceptualisations, il reste possible de considérer de manière isolée certaines distinctions fondamentales entre les différents sous-systèmes mnésiques. Ainsi, selon Cohen et Squire (Cohen \& Squire, 1980), il existe une différence fondamentale, à la fois sur le plan des représentations et sur celui des processus d'encodage et de récupération, entre la mémoire déclarative et la mémoire procédurale (voir figure 1).

Sur le plan cognitif, la mémoire déclarative est impliquée dans l'apprentissage, l'encodage et l'utilisation des connaissances relatives aux faits et aux événements et recouvre ainsi respectivement la mémoire sémantique et la mémoire épisodique (Eichenbaum \& Cohen, 2001; Squire \& Knowlton, 2000). Ces deux systèmes spécialisés sont dits explicites puisque les informations qui y sont encodées sont verbalisables et accessibles à la conscience (voir figure 1). En mémoire sémantique, ces informations ou concepts consistent en représentations symboliques des connaissances sur le monde qui nous entoure (ex., caractéristiques physiques et fonctionnelles des objets, sens des concepts abstraits, etc.), indépendantes de leur contexte d'acquisition (Caramazza, 2000; Samson, 2003). Par opposition, la mémoire épisodique (aussi appelée mémoire autobiographique) permet plutôt l'encodage à long terme des souvenirs personnels, soit des événements vécus par l'individu, situés dans leur contexte temporel et spatial d'acquisition (Piolino, 2003; Tulving, 2002). 


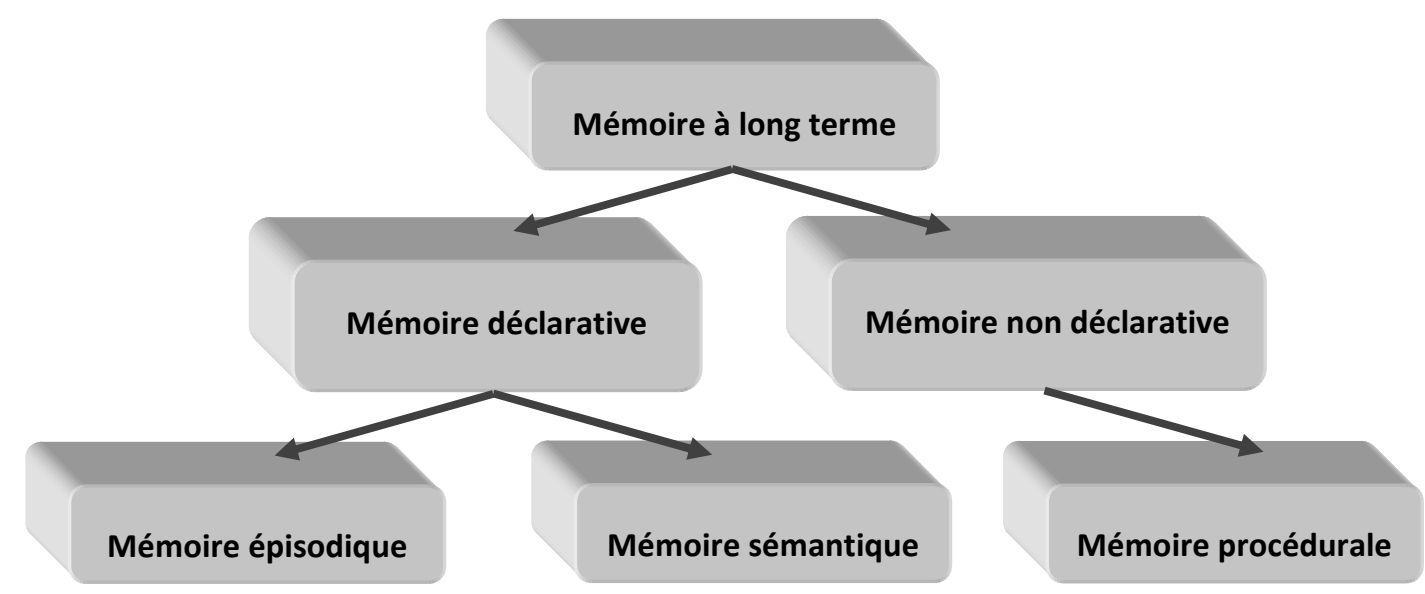

La mémoire procédurale est un système mnésique spécialisé pour l'apprentissage, le stockage et la récupération de procédures perceptuelles, sensori-motrices et cognitives (Eichenbaum \& Cohen, 2001; Squire \& Knowlton, 2000). Cette mémoire est par exemple impliquée dans l'acquisition et le maintien d'habiletés telles que rouler à bicyclette, utiliser un photocopieur, etc. La mémoire procédurale est dite "implicite" puisque, contrairement à la mémoire déclarative, l'apprentissage des connaissances et les connaissances elles-mêmes sont difficilement verbalisables et ne sont pas accessibles à la conscience.

\section{Mémoire à long terme et langage}

Suggérant une vision 'neurocognitive' de l'étude du lexique et de la grammaire, Ullman et ses collaborateurs (Ullman, 2001, 2004; Ullman \& Corkin, 1997; Ullman \& Pierpont, 2005) proposent un modèle intégratif, le modèle déclaratif/procédural (DP), dans lequel le lexique mental fait partie intégrante de la mémoire déclarative, alors que les processus en cause dans la grammaire mentale sont sous-tendus par la mémoire procédurale (voir figure 2).

De façon intrigante, les structures cérébrales (régions temporales médianes) dont dépend la mémoire déclarative sont identiques à celles dont relève le lexique mental (Damasio, Grabowski, Tranel, Hichwa, \& Damasio, 1996) et un parallèle identique peut aussi être fait sur le plan neuroanatomique entre la mémoire procédurale et la grammaire mentale, toutes deux sous-tendues corticalement par les ganglions de la base et les structures frontales (Grossman, 1999).

Les appuis au modèle DP émanent principalement de travaux menés en neuroimagerie (Friederici, 2002) ainsi qu'auprès de populations présentant des troubles développementaux du langage (Ullman \& Pierpont, 2005), une aphasie (Dronkers, Redfern, \& Knight, 2000) ou d'autres formes d'atteintes neurologiques comme la maladie d'Alzheimer, la maladie de Parkinson (MP) ou la maladie de Huntington (MH) (Ullman, 2004; Ullman \& Corkin, 1997). 
Figure 2. Représentation schématique de l'interrelation entre la mémoire à long terme et le langage.

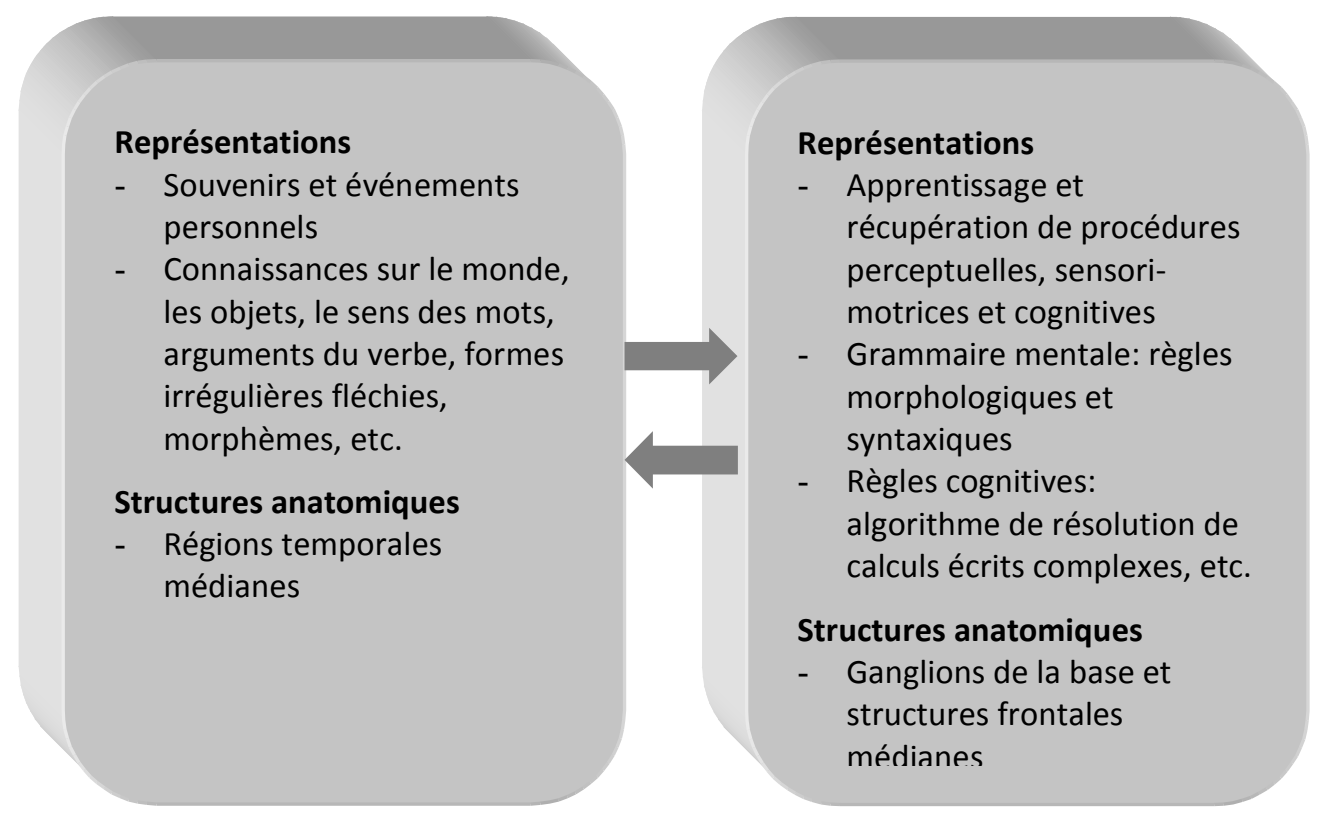

Ainsi, l'hypothèse selon laquelle les structures fronto-striatales (atteintes dans la MP et dans la MH) sont impliquées dans l'usage des règles grammaticales, tandis que les structures temporales (atteintes dans la maladie d'Alzheimer sont impliquées dans la récupération d'informations lexicales stockées en mémoire déclarative, a récemment été testée dans le domaine de la morphologie verbale (Price, 2006; Ullman \& Corkin, 1997). Les résultats indiquent que, contrairement au patients affectés par la maladie d'Alzheimer, qui présentent surtout des difficultés dans la production des formes irrégulières de certains verbes (ex : to dig : *dag au lieu de dug), les patients MP et MH présentent le patron inverse. Plus spécifiquement, la performance des patients MP est principalement affectée dans la production des formes conjuguées régulières ou 'nouvelles' (i.e. conjugaison de 'non-verbes') (ex., au passé : to look : *look ; to plag : *plag). Chez les patients $\mathrm{MH}$, on observe plutôt une tendance à la sur-régularisation (i.e., application erronée de la règle morphologique des verbes réguliers; ex., to dig :*digged) et à la sur-application de règles morphologiques (i.e., double application de la règle flexionnelle; ex., to look : *lookeded).

La dissociation dans les processus en cause en morphologie flexionnelle a également été explorée (Ullman et al., 2005) dans un groupe de 20 patients aphasiques post AVC comprenant 11 aphasiques non fluents agrammatiques avec lésion frontale, parfois associée à une lésion des régions temporale ou temporo-pariétale, et 9 aphasiques fluents avec atteinte temporale ou temporo-pariétale gauche. Soumis à trois tâches expérimentales de conjugaison au passé, de lecture à voix haute et de jugement de conjugaison portant sur des verbes réguliers (ex., look - looked) et irréguliers (ex., dig - dug), la performance de ces deux types de patients est très différente, même lorsque les facteurs d'influence tels que la fréquence, la complexité phonologique et les difficultés articulatoires sont contrôlés. Ainsi, alors que les aphasiques non fluents sont plus affectés pour les verbes réguliers que pour les verbes irréguliers, un patron inverse est plutôt relevé chez les aphasiques fluents. Ces résultats appuient donc les propositions du modèle DP selon lesquelles les structures temporo-pariétales sous-tendent le lexique mental tandis que la grammaire mentale, et en particulier le traitement des formes morphologiques régulières, est plutôt représentée corticalement dans les structures frontales.

Ces résultats sont importants aussi bien pour les théories linguistiques et psycholinguistiques que pour l'étude des fonctions cérébrales. Toutefois, étant restreints au seul domaine de la morphologie verbale, ils ne suffisent pas pour tester la pleine généralisation de l'hypothèse déclarative/procédurale. 
Dans une tentative d'étendre la recherche à d'autres domaines, une étude récente, menée en français auprès de personnes affectées par la MH (Teichmann et al., 2005), a exploré les processus d'application de règles dans le domaine de la morphologie verbale ainsi qu'en syntaxe et dans un domaine non linguistique, le calcul arithmétique. Alors que les patients $\mathrm{MH}$ ne présentent aucun trouble de nature déclarative, leur performance est déficitaire dès lors que l'application de règles est requise: 1) en morphologie, où ils ont tendance soit à sur-régulariser la conjugaison de non-verbes sous-réguliers en "ir" (ex., application de la règle morphologique du futur des verbes réguliers; saurentir : au futur :*il saurentera au lieu de il saurentira), soit à sur-appliquer les règles (ex., application de la règle morphologique du futur des verbes réguliers et des verbes sous-réguliers; saurentir : au futur : *il saurentirera); 2) en syntaxe, lorsqu'ils doivent juger de l'adéquation à une image de phrases «noncanoniques » (ex : les phrases passives) dans lesquelles les constituants ont été déplacés de leur position initiale (Chomsky, 1981) et où l'application de règles syntaxiques est nécessaire pour pouvoir correctement attribuer les rôles thématiques aux constituants déplacés (ex : dans la phrase passive : le garçon $_{\mathrm{i}}$ est poursuivi $\left(\mathrm{t}_{\mathrm{i}}\right)$ par le cheval, l'application de la règle syntaxique du passif permet de co-indexer la trace (t) avec le nom 'garçon' qui a été déplacé de sa position original 'objet' en position 'sujet'); 3) en calcul arithmétique écrit, lorsqu'ils doivent appliquer une règle d'emprunt dans les tâches de soustractions (ex., dans la soustraction 325 - 243, une règle d'emprunt doit être appliquée puisque le nombre de dizaines du ler nombre (2) n'est pas suffisant pour retrancher directement le nombre de dizaines du second (4) et requiert alors l'emprunt d'une unité au rang suivant).

\section{Différenciation dans l'application des règles procédurales: exploration à partir d'un cas unique}

Dans le but explicite d'identifier de possibles différences de traitement cognitif selon la nature des règles procédurales dans les domaines linguistique et non linguistique, nous avons récemment effectué une étude de cas unique auprès d'un patient, Mr FG, âgé de 74 ans, qui a subitement présenté des troubles du langage à la suite d'un AVC gauche. Dans cette section, nous présentons brièvement cette étude de manière à illustrer l'importance de l'interrelation entre la mémoire procédurale et les capacités linguistiques.

\subsection{Résumé de l'histoire de cas}

FG est un homme de 74 ans, droitier, francophone, dont le niveau de scolarité correspond à 11 ans. Il nous est référé en juillet 2005 lors d'une hospitalisation relative à l'exacerbation d'une maladie bipolaire. Le rapport médical mentionne l'apparition subite, en janvier 2003, d'un syndrome d'accent étranger accompagné d'agrammatisme mais pour lesquelles aucune évaluation ni prise en charge thérapeutique n'avaient été effectuées en orthophonie (Poulin, Macoir, Paquet, Fossard, \& Gagnon, 2007).

Sur le plan cognitif, l'évaluation neuropsychologique rend compte de la présence de déficits de la mémoire de travail et des fonctions exécutives (inhibition, flexibilité et planification) alors que la performance du patient est normale dans toutes les tâches explorant l'orientation, l'attention, les gnosies visuelles, les praxies et la mémoire épisodique

Sur le plan du langage, on note la présence d'un léger agrammatisme marqué par la l'omission de mots fonctions et de morphèmes grammaticaux ainsi que par la production de structures syntaxiques appauvries. FG présente également la plupart des caractéristiques segmentales et suprasegmentales habituellement rapportées pour le syndrome d'accent étranger. Par ailleurs, sa performance est normale dans les tâches de compréhension orale et écrite, de répétition et de dénomination, alors qu'on observe une légère dyslexie phonologique et un déficit dans les tâches de fluidité verbale, probablement attribuable à l'atteinte des fonctions exécutives.

Sur le plan radiologique, l'IRM et l'examen TEP rendent compte d'une atrophie asymétrique dans le lobe temporal et la région de l'insula gauche (Poulin et al., 2007). 


\subsection{Résumé de l'étude expérimentale}

FG a été soumis à une batterie expérimentale destinée à explorer les processus en cause en mémoire déclarative et en mémoire procédurale dans deux domaines linguistiques, la morphologie et la syntaxe, ainsi que dans un domaine non linguistique, le calcul arithmétique (Macoir, Fossard, Nespoulous, Demonet \& Bachoud-Lévi, soumis). En plus du domaine d'application, la nature même des règles a été prise en considération dans les diverses tâches expérimentale. La performance du patient confirme bien la différenciation entre mémoire déclarative (préservée) et mémoire procédurale (affectée) et vient de plus, questionner la nature et la spécificité des règles sous-tendues par cette dernière.

\subsubsection{Mémoire procédurale et processus morphologiques flexionnels}

En morphologie flexionnelle, FG ne présente aucune difficulté à conjuguer (infinitif au présent et vice versa; présent au futur et vice versa) les verbes réguliers (ex., manger: 72/72, 100\%) et sous-réguliers (ex., sortir: 72/72, 100\%). Par contre, le patient présente d'importantes difficultés lorsqu'il doit effectuer les mêmes tâches avec des non-verbes (ex., non-verbes réguliers: FG =15/48, 31\%; sujets contrôles = 48/48, 100\%: ex., conjuguer au présent le non-verbe pover: *il pova au lieu de "il povera") (voir Tableau 1).

Tableau 1. Performance de FG et des sujets contrôles (nombre et pourcentage de bonnes réponses; les valeurs entre parenthèses correspondent à l'écart-type) dans la tâche de flexion de non-verbes.

\begin{tabular}{ccccc}
\hline Tâches de conjugaison & \multicolumn{2}{c}{ Non-verbes réguliers } & \multicolumn{2}{c}{ Non-verbes sous-réguliers } \\
& FG & Contrôles & FG & Contrôles \\
Infinitif $\leftrightarrow$ présent $(24)$ & $24(100 \%)$ & $23.75[.05](99 \%)$ & $18(75 \%)$ & $22.25[.16](96 \%)$ \\
Présent $\leftrightarrow$ futur $(24)$ & $14(58 \%)$ & $24(100 \%)^{* * *}$ & $13(54 \%)$ & $21[.29](87.5 \%)^{*}$ \\
Infinitif $\leftrightarrow$ futur $(24)$ & $1(4 \%)$ & $24(100 \%)^{* * *}$ & $24(100 \%)$ & $23.75[.05](99 \%)$ \\
\hline
\end{tabular}

Différence entre FG et les contrôles: $* \mathrm{p}<.05 ; * * * \mathrm{p} \leq .001$

Sa performance est également bien préservée quand il est soumis à une tâche d'accord en genre d'adjectifs réguliers, ayant une forme identique au masculin et au féminin (ex., modeste: 10/10, 100\%) et sousréguliers, dont la forme varie selon le genre (ex., petit: 20/20, 100\%). FG présente cependant d'importantes difficultés dans la tâche de flexion en genre des non-adjectifs (ex., non-adjectifs sousréguliers: $\mathrm{FG}=19 / 40,47.5 \%$; sujets contrôles $=36.25 / 40,91 \%$ : ex., produire au féminin le non-adjectif il est oubreux: *elle est oubreux au lieu de "elle est oubreuse") (voir Tableau 2).

Tableau 2. Performance de FG et des sujets contrôles (nombre et pourecentage de bonnes réponses) dans la tâche de flexion de non-adjectifs

\begin{tabular}{ccccc}
\hline Tâches de flexion & \multicolumn{2}{c}{ Non-adjectif sans règle (10) } & \multicolumn{2}{c}{ Non-adjectif avec règle (20) } \\
& FG & Contrôles & FG & Contrôles \\
Masculin $\leftrightarrow$ féminin & $9(90 \%)$ & $10(100 \%)$ & $13(65 \%)$ & $20(100 \%)^{* *}$ \\
Féminin $\leftrightarrow$ masculin & $10(100 \%)$ & $\begin{array}{l}9.75[.5] \\
(97.5 \%)\end{array}$ & $6(30 \%)$ & $17\left[3.16(85 \%)^{* *}\right.$ \\
\hline
\end{tabular}

Différence entre FG et les contrôles: $* * p<.01$ 
La bonne préservation de la mémoire déclarative permet ainsi à FG de compenser son déficit procédural et d'effectuer adéquatement les opérations flexionnelles des verbes et des adjectifs. Lorsque le recours à cette mémoire déclarative n'est pas possible comme dans le cas de la flexion des non-verbes et des nonadjectifs, le déficit procédural entraîne chez lui d'importantes difficultés, surtout lorsque la tâche requiert l'application de plusieurs règles spécifiques (ex., la conjugaison du verbe manger de la 3ème personne du singulier du présent à la 3ème personne du singulier du futur requiert l'application de 2 règles flexionnelles spécifiques: ajouter l'affixe du futur -er et ajouter l'affixe de la personne -a).

\subsubsection{Mémoire procédurale et processus syntaxiques}

En syntaxe, soumis à une tâche d'anagramme, FG ne présente aucune difficulté $(\mathrm{FG}=31 / 32,97 \%$; sujets contrôles $=32 / 32,100 \%$ ) à replacer dans l'ordre correct les mots de phrases 'nominalisées' actives à 2 ou 3 arguments (ex., la fillette confie un secret à ses amis) qui ne requièrent que l'application d'une règle générale consistant à placer les arguments du verbe selon l'ordre canonique en français (Sujet-VerbeObjet).

Tableau 3: Performance de FG et des sujets contrôles (nombre et pourcentage de bonnes réponses; les valeurs entre parenthèses correspondent à l'écart-type) dans la tâche d'anagramme.

\begin{tabular}{|ccccc|}
\hline & Nom. active \#1 & Nom. active \#2 & Pronom. active \#1 & Pronom. active \#2 \\
FG & $16 / 16(100 \%)$ & $15 / 16(94 \%)$ & $14 / 16(87.5 \%)$ & $8 / 16^{* *}(50 \%)$ \\
Contrôles & $16[0](100 \%)$ & $16[0](100 \%)$ & $16[0](100 \%)$ & $15.75[0.2](98 \%)$ \\
\hline - & Phrases nominalisées actives \#1: le garçon creuse un trou avec ses parents \\
- & Phrases pronominalisées actives \#1: il le creuse avec eux \\
- & Phrases nominalisées actives \#2: la fillette confie un secret à ses amies \\
- & Phrases pronominalisées actives \#1: elle le leur confie
\end{tabular}

Différence entre FG et les contrôles: ** $\mathrm{p}<.01$

Par contre, il présente d'importantes difficultés $(\mathrm{FG}=22 / 32,69 \%$; sujets contrôles $=31.75 / 32,99 \%$ ) lorsque les versions pronominalisées des mêmes phrases sont utilisées. Dans ce cas particulier, il applique correctement la règle générale selon laquelle les pronoms clitiques se placent devant le verbe, mais ne peut appliquer la règle d'agencement spécifique de ces pronoms (ex : *elle leur le confie au lieu de "elle le leur confie") (voir Tableau 3)

\subsubsection{Mémoire procédurale et traitement numérique}

Le déficit procédural du patient est également relevé dans les tâches de calcul arithmétique. Dans ce domaine non linguistique, la performance de FG est excellente (ex., FG = 11/12 pour les multiplications; sujets contrôles $=11 / 12$, E-T = .22) dans les tâches de calcul écrit (ex., 134 x 22) qui, en plus de la récupération en mémoire déclarative des faits arithmétiques (ex., 2 x $4=8$ ), requièrent l'application d'une règle générale ou algorithme de calcul (ex., commencer à la colonne située à l'extrême droite; récupérer le produit des chiffres qui y figurent; écrire le chiffre correspondant au bas de la colonne; etc.) qui s'applique à toutes les occurrences de la même opération.

FG rencontre cependant d'importantes difficultés dans les tâches de calcul mental. En effet, alors qu'il récupère parfaitement (ex., 45/45 pour les soustractions) les faits arithmétiques en mémoire déclarative, il parvient difficilement à effectuer les calculs mentaux qui requièrent l'application de règles spécifiques comme c'est le cas pour $\mathrm{n}$ x $0=0(\mathrm{FG}=0 / 9$, sujets contrôles $=8.67 / 12, \mathrm{E}-\mathrm{T}=.19)$ ou pour $\mathrm{n} \div \mathrm{n}=1(\mathrm{FG}$ $=1 / 20$, sujets contrôles $=14.66 / 20, \mathrm{E}-\mathrm{T}=.28)($ voir Tableau 4$)$. 
Tableau 4. Performance de FG et des sujets contrôles (nombre et pourcentage de bonnes réponses; les valeurs entre parenthèses correspondent à l'écart-type) dans les tâches de calcul simple.

\begin{tabular}{rrr}
\hline Tâches de calcul & FG & Contrôles \\
Calcul simple sans règle & & \\
$-\quad$ Additions (60) & $59(98 \%)$ & $58.751 .25](98 \%)$ \\
$-\quad$ Soustractions (45) & $45(100 \%)$ & $44.5[.5](99 \%)$ \\
$-\quad$ Multiplications (81) & $77(95 \%)$ & $80.5[.06](99 \%)$ \\
$-\quad$ Divisions (27) & $23(85 \%)$ & $27(100 \%)$ \\
Calcul simple avec règle & & \\
$-\quad 0 \div \mathrm{n}=0(9)$ & $0(0 \%)$ & $14.66[.17](89 \%) * * *$ \\
$-\quad \mathrm{n} \div \mathrm{n}=1(20)$ & $1(5 \%)$ & $8.67[.19](96 \%) * * *$ \\
$-\quad \mathrm{n} \times 0 * 0(9)$ & $0(0 \%)$ & $9(100 \%) * * *$ \\
\hline
\end{tabular}

Différence entre FG et les contrôles: *** $\mathrm{p}<.001$

\subsection{Conclusions de l'étude expérimentale}

De manière générale, ces résultats appuient le modèle DP (S. Pinker, 1999; Ullman, 2001) et la distinction en cognition entre d'une part l'activation des représentations encodées dans la mémoire déclarative et l'application de règles en mémoire procédurale d'autre part.

L'originalité de l'étude réside essentiellement dans l'exploration de différences de traitement cognitif selon la nature des règles procédurales. En effet, selon les résultats de cette étude, il semble qu'il soit possible de différencier les règles en mémoire procédurale et ainsi distinguer l'application de règles générales, s'appliquant à tous les cas d'un domaine, de l'application de règles spécifiques, restrictives à des cas particuliers du domaine (Macoir et al., soumis). Cette tentative de différenciation devra cependant être explorée plus avant de manière à préciser l'influence respective des facteurs de complexité, de nombre et de spécificité des règles sur le traitement procédural.

\section{Conclusions générales}

Comme en témoignent ces études, le langage n'est pas une fonction cognitive isolée des autres fonctions mentales supérieures. Ses liens avec la mémoire sémantique, les fonctions exécutives, la mémoire de travail sont de plus en plus reconnus. Plusieurs travaux récents tendent également à démontrer que le langage est fortement associé, sur le plan fonctionnel et neuroanatomique, avec les capacités de la mémoire procédurale. S'il est communément accepté de considérer que les différents mots et morphèmes encodés dans le lexique mental correspondent en fait à des représentations dans la mémoire déclarative, la conceptualisation des processus grammaticaux sous l'angle de l'activation de règles en mémoire procédurale est beaucoup plus récente.

Les travaux rapportés dans cet article, ainsi que l'étude de cas unique que nous avons récemment menée, confirment l'importance de considérer de façon distincte les capacités qui relèvent de la mémoire déclarative et de la mémoire procédurale.

Sur le plan clinique, l'identification précise d'une atteinte fonctionnelle de la mémoire procédurale pourrait par exemple mener à l'établissement d'un plan de traitement individualisé visant le rétablissement ou le réapprentissage explicite des procédures d'application de règles. A titre d'exemple, une fois identifiée la nature procédurale d'un trouble morphologique flexionnel, l'administration d'une stratégie rééducative de rétablissement de l'application des règles morphologiques affectées pourrait être envisagée 
au moyen de tâches de conjugaison de verbes puis de non-verbes, dans lesquelles les règles flexionnelles de complexité croissante seraient progressivement introduites de manière explicite.

\section{Références bibliographiques}

Caramazza, A. (2000). The organization of conceptual knowledge in the brain. In M.S. Gazzaniga (Ed): The Cognitive Neurosciences, $2^{\text {nd }}$ ed. Cambridge: MIT Press, 1037-1046.

Chomsky, N. (1981). Lectures on government and binding. Dordrecht: Foris.

Chomsky, N. (1995). The minimalist program. Cambridge, MA: MIT Press.

Cohen, N., \& Squire, L. (1980). Preserved learning and retention of pattern-analyzing skill in amnesia: dissociation of knowing how and knowing that. Science, 210 (4466), 207-210.

Damasio, H., Grabowski, T., Tranel, D., Hichwa, R., \& Damasio, A. (1996). A neural basis for lexical retrieval. Nature, 380, 499-505.

Dronkers, N. F., Redfern, B. B., \& Knight, R. T. (2000). The neural architecture of language disorders. In M. S. Gazzaniga (Ed.): The new cognitive neurosciences. Cambridge: MIT Press, 949-958

Eichenbaum, H., \& Cohen, J. (2001). From conditioning to conscious recollection: memory systems of the brain. New York: Oxford University Press.

Eustache, F., \& Desgranges, B. (2003). Concepts et modèles en neuropsychologie de la mémoire: entre théorie et pratique clinique. In T. Meulemans, B. Desgranges, S. Adam \& F. Eustache (Eds): Évaluation et prise en charge des troubles mnésiques. Marseille: Solal, 13-49.

Friederici, A. D. (2002). Towards a neural basis of auditory sentence processing. Trends in Cognitive Sciences, 6 (2), 78-84.

Gazzaniga, M. S., Ivy, R. B., \& Mangun, G. R. (2002). Cognitive neuroscience: The biology of mind, $2^{\text {nd }}$ ed. New York: W.W. Norton \& Company.

Grossman, M. (1999). Sentence processing in Parkinson's disease. Brain and Cognition, 40, 387-413.

Macoir, J., Fossard, M., Nespoulous, J.-L., Demonet, J.-F., \& Bachoud-Levi, A. C. (soumis). Selective deficit of rule application in morphology, syntax and number processing: Procedural or working memory deficit?

Marcus, G. F. (2000). The algebric mind: Reflections on connectionism and cognitive science. Cambridge: MIT Press.

Marslen-Wilson, W., \& Tyler, L. K. (1997). Dissociating types of mental computation. Nature, 387, 592-594.

Pinker, S. (1999). Words and rules: The ingredients of language. London: Weidenfeld \& Nicolson.

Pinker, S., Jackendoff, R. (2005). The faculty of language: What's special about it? Cognition, 95, 201-236.

Piolino, P. (2003). La mémoire autobiographique: modèles et évaluation. In T. Meulemans, B. Desgranges, S. Adam \& F. Eustache (Eds): Évaluation et prise en charge des troubles mnésiques. Marseille: Solal, 195-221.

Poulin, S., Macoir, J., Paquet, N., Fossard, M., \& Gagnon, L. (2007). Psychogenic or neurogenic origin of agrammatism and foreign accent syndrome in a bipolar patient: a case report. Annals of General Psychiatry, 6, 1 .

Price, A. L. (2006). Explicit category learning in Parkinson's disease: deficits related to impaired rule generation and selection processes. Neuropsychology, 20 (2), 249-257.

Samson, D. (2003). La mémoire sémantique: modèles et évaluation. In T. Meulemans, B. Desgranges, S. Adam \& F. Eustache (Eds): Évaluation et prise en charge des troubles mnésiques. Marseille: Solal, 169-193.

Schacter, D. L., Wagner, A. D., \& Buckner, R. L. (2000). Memory systems of 1999. In E. Tulving \& F. I. M. Craik (Eds): The Oxford handbook of memory. New York: Oxford University Press, 627-643.

Squire, L. R., \& Knowlton, B. J. (2000). The medial temporal lobe, the hippocampus, and the memory system of the brain. In M. S. Gazzaniga (Ed.): The new cognitive neurosciences. Cambridge: MIT Press, 765-780. 
Squire, L. R., Stark, C. E., \& Clark, R. E. (2004). The medial temporal lobe. Annual Review of Neuroscience, 27, 279-306.

Teichmann, M., Dupoux, E., Kouider, S., Brugieres, P., Boisse, M. F., Baudic, S., et al. (2005). The role of the striatum in rule application: the model of Huntington's disease at early stage. Brain, 128 (Pt 5), 1155-1167.

Tulving, E. (2002). Episodic memory: From Mind to Brain. Annual Review of Psychology, 53 (1), 1-25.

Ullman, M. T. (2001). The declarative/procedural model of lexicon and grammar. Journal of Psycholinguistic Research, 30 (1), 37-69.

Ullman, M. T. (2004). Contributions of memory circuits to language: the declarative/procedural model. Cognition, 92 (1-2), 231-270.

Ullman, M. T., \& Corkin, S. (1997). A neural dissociation within language: Evidence that the mental dictionary is part of declarative memory, and that grammatical rules are processed by the procedural system. Journal of Cognitive Neuroscience, 9 (2), 266-276.

Ullman, M. T., Pancheva, R., Love, T., Yee, E., Swinney, D., \& Hickok, G. (2005). Neural correlates of lexicon and grammar: evidence from the production, reading, and judgment of inflection in aphasia. Brain and Language, 93 (2), 185-238

Ullman, M. T., \& Pierpont, E. I. (2005). Specific language impairment is not specific to language: the procedural deficit hypothesis. Cortex, 41(3), 399-433. 